\title{
Mature cystic teratoma of the fallopian tube associated with uterine adenomyoma in a postmenopausal woman
}

\author{
Sunil Kumar Samal*, Setu Rathod, Reddi Rani
}

Department of Obstetrics and Gynecology, Mahatma Gandhi Medical College and Research Institute, Sri Balaji Vidyapeeth,

(Deemed to be University),

Pillaiyarkuppam, Puducherry-607402,

For Correspondence

*Dr. Sunil Kumar Samal,

Email: sunilkumars@mgmcri.ac.in

Date of

Submisssion: 15-11-2018

Acceptance: 29-11-2018
Access this article online

Quick Response Code

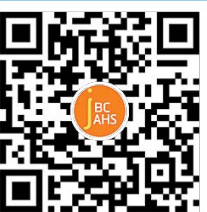

https://www.jbcahs.org

\section{ABSTRACT}

Teratoma of the fallopian tube (solid or cystic) is extremely uncommon. In addition, the incidence of a mature cystic teratoma of fallopian tube containing haemorrhagic fluid in association with uterine adenomyoma is extreme low. In the current case report, we present a case of mature cystic teratoma of the fallopian tube containing haemorrhagic fluid with uterine adenomyoma in a 46 year old female who attended menopause one year back. The patient underwent total abdominal hysterectomy and bilateral salpingooophorectomy. Histopathology confirmed the diagnosis of fallopian tube teratoma.

\section{Keywords:}

mature cystic teratoma, fallopian tube, adenomyoma

\section{CASE REPORT}

We report a case of 46-year lady P1L1 who attended menopause one year back presented with heavy bleeding per vaginum for last 7 days. After evaluation she was diagnosed as a case of adenomyosis of uterus with left ovarian simple cyst of size $4 \times 4 \mathrm{~cm}$. The patient had undergone abdominal hysterectomy with bilateral salpingo oophorectomy. Intraoperatively, along with adenomyosis, a haemorrahagic cyst (Figure 1) of size $4 \times 4 \mathrm{~cm}$ was found at the infundibular part of the left fallopian tube. Bilateral ovaries were normal. Cross section (Figure 2) of cyst revealed, $20 \mathrm{ml}$ of haemorrhagic fluid with a tooth like structure in the wall. Histopathology confirmed the diagnosis of mature teratoma of the fallopian tube. The patient had no symptoms or signs at the 6-month follow-up.

\section{DISCUSSION}

The first case of fallopian tube teratoma was reported by Eden and Lockyer in 1865. ${ }^{1}$ From then 74 cases has been reported in literature including 5 bilateral cases and 3 cases of immature teratoma. ${ }^{1,2}$ In most of the cases of cystic teratomas of the fallopian tube, the most common sites were isthmus or ampullary regions ${ }^{2,3}$ like in our case where it was ampullary region. In majority of the cases, the anatomical location of the teratoma slightly prevalent in the right tube and is usually intraluminal and rarely intramural or subserosal. ${ }^{2,3}$ The reported cases were either nullipara or have parity of less than two like in our case where she was primipara. Majority of the reported cases were in their 40s, while in our case she was post-menopausal. In literature none of the reported cases were diagnosed preoperatively as like our case where the finding of dermoid cyst was incidental during examination of specimen. Rupture of cyst with signs of peritonitis has been reported in one case.

It is hypothesized that initially teratomas contain abundant mesenchymal stroma, but eventually develops both endodermal and ectodermal derivatives with bronchial tissues, thyroid, brain and skin appendages. In our case, the
How to Cite: Sunil Kumar Samal, Setu Rathod, Reddi Rani, Mature Cystic Teratoma of the Fallopian tube associated with Uterine Adenomyoma in a postmenopausal woman. J Basic Clin Appl Health Sci. 2018;1:49-50 
Figure 1: Fallopian tube dermoid with adenomyosis of uterus

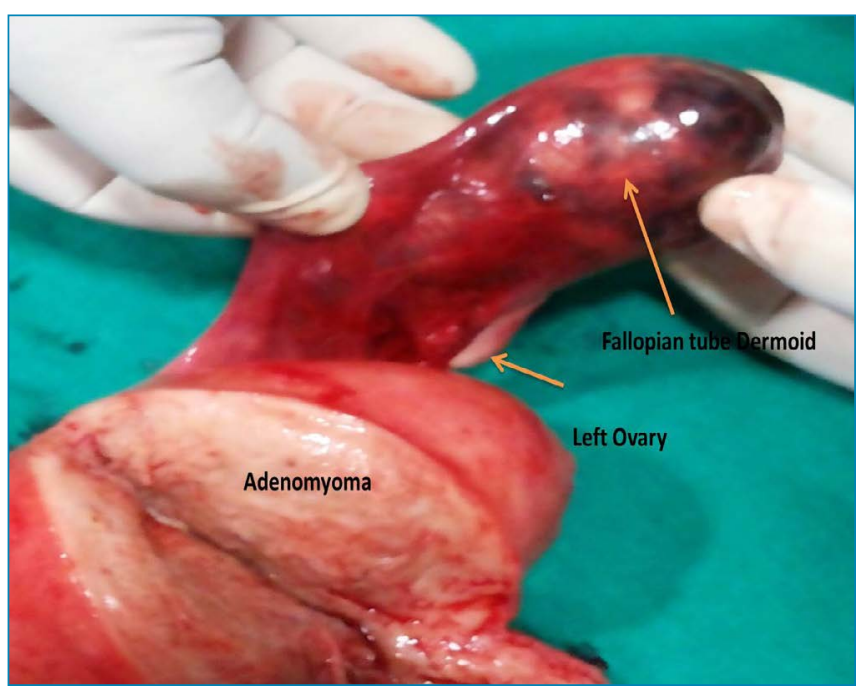

contains of the teratoma were mostly mesodermal in origin. In literature, the clinical presentations of the reported cases varies from infertility, abnormal uterine bleeding, lower abdominal pain, pain in the iliac region, symptoms of acute appendicitis or endometriosis, intrauterine pregnancy, hemoperitoneum, and/or ectopic pregnancy. A case of solid teratoma in the intraluminal location of the fallopian tube associated with intrauterine leiomyomas has been reported. ${ }^{3}$

\section{CONCLUSION AND CLINICAL SIGNIFICANCE}

Fallopian tube teratoma associated with adenomyosis is extremely rare. The prognosis is favourable following
Figure 2: Cut section of dermoid showing tooth like structure

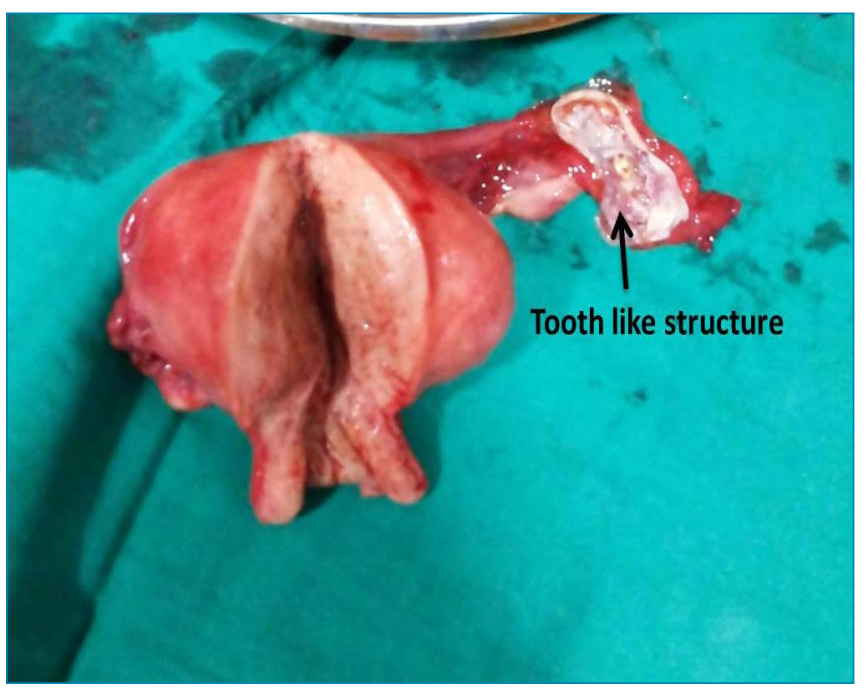

complete surgical excision.Gynaecologist should consider the possibility of this disease entity while making differential diagnosis.

\section{References}

1. Roncati L, Barbolini G, Ghirardini G, Rivasi F. Mature solid teratoma of the fallopian tube mimicking metastasis of endometrial adenocarcinoma: a case report.Int J Surg Pathol. 2010; 18:561-3

2. Khatib G, Guzel AB, Kucukgoz-Gulec U, Vardar MA, Musaev A, Melekoglu R. Mature cystic teratoma of the fallopian tube. J Obstet Gynaecol. 2013;33:120-4

3. Chao TJ, Chao J, Kuan LJ, Li YT, Kuo TC, Chang YC et al. Mature solid teratoma of the fallopian tube associated with uterine leiomyomas. J Chin Med Assoc. 2008;71:425-7.

\section{CAR T-cell Therapy: A New Era in Cancer Immunotherapy}

Chimeric Antigen Receptor (CAR) T-cell therapy involves genetic modification of patient's autologous T-cells to express a CAR specific for a tumor antigen, following by ex vivo cell expansion and re-infusion back to the patient. CARs are fusion proteins of a selected single-chain fragment variable from a specific monoclonal antibody and one or more T-cell receptor intracellular signaling domains. This T-cell genetic modification may occur either via viralbased gene transfer methods or nonviral methods, such as DNA-based transposons, CRISPR/Cas9 technology or direct transfer of in vitro transcribed-mRNA by electroporation. Clinical trials have shown very promising results in end-stage patients with a full recovery of up to $92 \%$ in Acute Lymphocytic Leukemia. Despite such results in hematological cancers, the effective translation of CAR T-cell therapy to solid tumors and the corresponding clinical experience is limited due to therapeutic barriers, like CAR T-cell expansion, persistence, trafficking, and fate within tumors.

Source: Curr Pharm Biotechnol. 2018;19(1):5-18 